\title{
Defects in Two Dimensional Crystals: An Ultra-high Resolution Aberration-corrected Electron Microscopy Study
}

N. Alem ${ }^{1}$, Q.M. Ramasse ${ }^{2}$, O.V. Yazyev ${ }^{3}$, C.R. Seabourne ${ }^{4}$, C.K. Kisielowski ${ }^{5}$, P. Hartel ${ }^{6}$, B. Jiang ${ }^{7}$, R. Erni $^{8}$, K. Erickson ${ }^{9}$, M.C. Sarahan ${ }^{2}$, M.D. Rossell ${ }^{8}$, A.J. Scott ${ }^{4}$, S.G. Louie ${ }^{9}$, A. Zettl ${ }^{9}$

${ }^{1}$ Materials Science and Engineering Department, The Pennsylvania State University, University Park, PA, 16802, USA

${ }^{2}$ SuperSTEM Laboratory, STFC Daresbury, Keckwick Lane, Daresbury WA4 4AD, UK

${ }^{3}$ Institute of Theoretical Physics, École Polytechnique Fédérale de Lausanne (EPFL), CH-1015 Lausanne, Switzerland

${ }^{4}$ Institute for Materials Research, SPEME, University of Leeds, Leeds LS2 9JT, UK

${ }^{5}$ National Center for Electron microscopy, Lawrence Berkeley National Laboratory, California, USA

${ }^{6} \mathrm{CEOS} \mathrm{GmbH,} \mathrm{Englerstrasse} \mathrm{28,} \mathrm{D-69126} \mathrm{Heidelberg,} \mathrm{Germany}$

${ }^{7}$ FEI Company, NE Dawson Creek Drive, Hillsboro, Oregon 97124, USA

${ }^{8}$ Empa, Swiss Federal Laboratories for Materials Science and Technology, Switzerland

${ }^{9}$ Department of Physics, University of California - Berkeley, Berkeley CA94720, USA

Defects can modulate the electronic properties of crystals by introducing empty states within their band gap. When exposed to chemical functional groups, defects can trap molecules and adatoms and significantly change the electronic and sensing properties of the crystal. Atomically thin two dimensional crystals, such as graphene and hexagonal boron nitride (h-BN) are considered emerging materials with variety of applications in in sensing [1], hydrogen storage [2], and electronics [3]. Structural relaxations at the defects and edges in these crystals can have a significant impact on their resulting physical, chemical and electronic properties. This work presents the relaxation effects at the edges and vacancies at the sub-Angstrom size scales and its effect on the physical and electronic properties of h-BN.

In this study, we use ultra-high resolution aberration-corrected electron microscopy to probe the underlying physics at the atomic scale in a bilayer of h-BN. Our experimental investigations coupled with first-principles calculations uncover formation of interlayer bonds across the bilayer $h-B N$ membrane at the boron monovacancies and at the zigzag edges [4]. We probe the sub-Ångstrom structural distortions that occur at the defects in h-BN membrane using phase contrast imaging (Fig. 1). This structural distortion alters the scattering potential, leading to a significant phase shift around the boron monovacancies and edges. Such a phase shift leads to the chemical identification of boron monovacancies as opposed to nitrogen defects.

Large terraces and step edges mostly in the zigzag direction are formed across the h-BN as a result of sample beam interactions. Atomic column EELS analysis and $\mathrm{Z}$ contrast imaging coupled with $a b$ initio calculations reveal interlayer B-N bond formation across the zigzag edges in bilayer h-BN (Fig. 2), Similar to monovacancies. As a result of different electron beam channeling at the distorted edges, a higher intensity in $\mathrm{Z}$ contrast $\mathrm{ADF}$ images are observed. Such structural relaxations also lead to different electronic properties in h-BN [5]. 


\section{References:}

1. Ye Lu et al., Appl. Phys. Lett. 97, 083107 (2010)

2. Chen X. et al., J. Phys. Chem. B, 2005, 109 (23), pp 11525-11529

3. C. R. Dean et al., Nature Nanotechnology 5, 722-726 (2010)

4. N. Alem et al., Phys. Rev. Lett. 106, 126102 (2011)

5. N. Alem et al., Phys. Rev. Lett. 109, 205502 (2012)
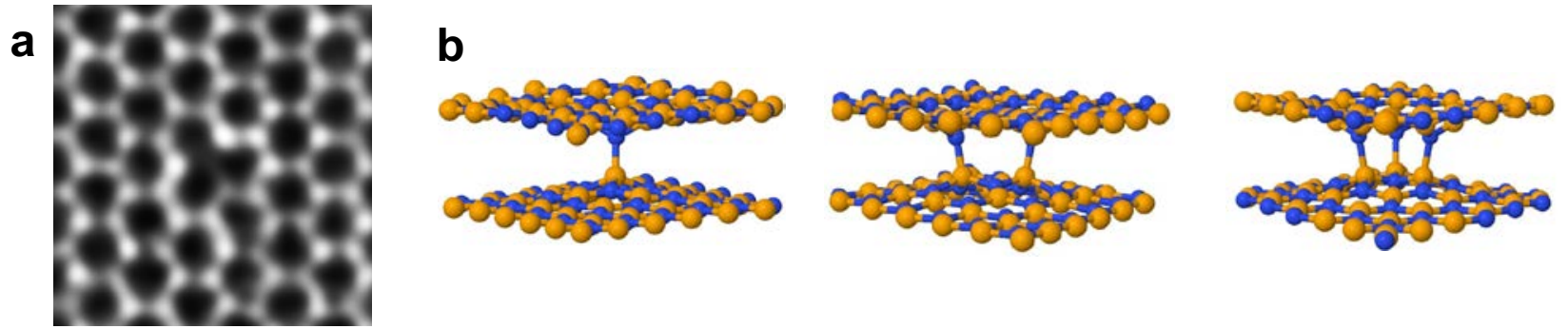

Fig 1. A boron monovacancy in a bilayer h-BN showing a higher phase shift at the edge atoms compared with the defect free region (a). DFT models showing the formation of B-N interlayer bonds locally forming at the vacancies across the bilayer.
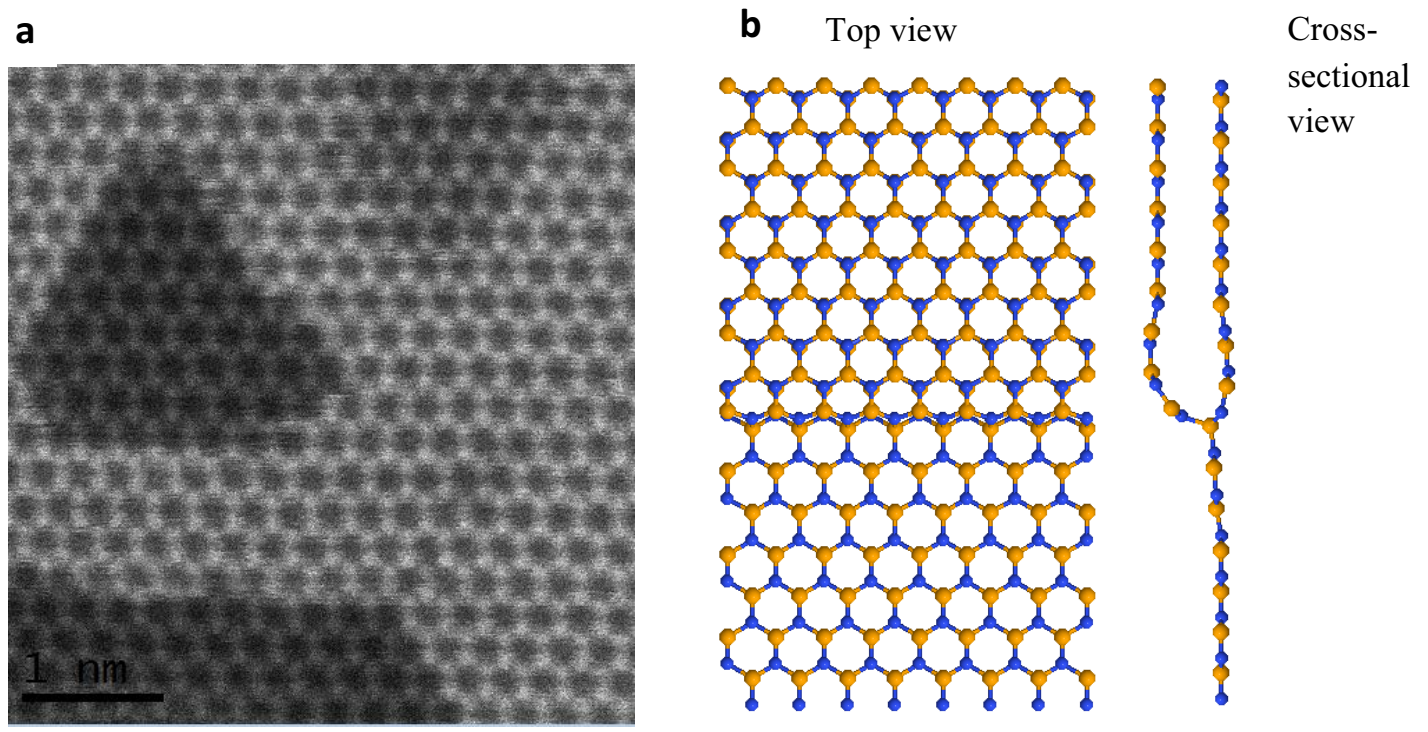

Fig 2. An annular dark field STEM image of atomically thin h-BN (a), showing step edges with zigzag configuration. Density functional theory calculations predict interlayer bonds at the edges of h-BN leading to the distortion of the film (b). 